\title{
Sexual Activation of Carotenogenesis in Phycomyces blakesleeanus
}

\author{
By N. S. GOVIND† AND E. CERDÁ-OLMEDO* \\ Departamento de Genética, Facultad de Biologia, Universidad de Sevilla, Apartado 1095, \\ E-41080 Sevilla, Spain
}

(Received 18 April 1986)

\begin{abstract}
Sexual stimulation increases the $\beta$-carotene content of the fungus Phycomyces blakesleeanus. The same effect is observed in single cultures exposed to natural and synthetic trisporates and in intersexual heterokaryons. Synthetic racemic $(9 Z)$-methyl trisporate B, a trisporate precursor made by cultures of the $(+)$ mating type, stimulates carotenogenesis only in $(-)$ cultures. Synthetic racemic $(9 Z)$-methyl trisporate $C$ is less effective and the corresponding all- $(E)$ isomers and other related compounds are inactive. Sexual stimulation of carotenogenesis is additive with the stimulations induced by light, retinol, dimethyl phthalate, and mutation of the gene carS.
\end{abstract}

\section{INTRODUCTION}

Several external stimuli accentuate the yellow colour of the fungus Phycomyces blakesleeanus by increasing its $\beta$-carotene content (review by Cerdá-Olmedo \& Torres-Martínez, 1979). The main active agents are light (Bergman et al., 1973; Jayaram et al., 1979; López-Díaz \& Cerdá-Olmedo, 1980), sexual interaction, and various chemicals, including $\beta$-ionone (Mackinney et al., 1952), retinol (Eslava et al., 1974), dimethyl phthalate and others (Cerdá-Olmedo \& Hüttermann, 1986).

There are several kinds of mutants with defective $\beta$-carotene synthesis. Some of them block specific reactions of the pathway: $c a r R$ mutants are red and accumulate lycopene, $\operatorname{car} B$ mutants are white and accumulate phytoene. A high constitutive level of $\beta$-carotene is found in $c a r S$ mutants. Most car $A$ mutants are white and contain only little $\beta$-carotene, but become yellow on addition of retinol. Mutations having little or no effect on the basal $\beta$-carotene content but rendering the mycelia insensitive to the action of retinol and other chemicals occur at genes carI and $\operatorname{car} A$. For references on these mutants see Table 1 and the review by Cerdá-Olmedo (1985).

There are two mating types or sexes in Phycomyces, called (+) and (-) (Blakeslee, 1904). Hyphae of opposite sex growing near each other have a pronounced yellow colour (Blakeslee, 1904; Hocking, 1965). Trisporic acids, found in mated cultures of the Mucorales, including Blakeslea trispora (Caglioti et al., 1966; Sutter \& Whitaker, 1981) and P. blakesleeanus (Sutter, 1977), stimulate carotene production and sexual morphogenesis when added to single cultures. Trisporic acids are made from $\beta$-carotene (Austin et al., 1969) via sex-specific precursors (Sutter et al., 1974). The methyl trisporates are produced by the $(+)$ mycelia and converted to trisporic acids by the $(-)$ mycelia (Sutter \& Whitaker, 1981). Intersexual heterokaryons, whose mycelia mix nuclei of both sexes (Burgeff, 1914), form many bizarre morphological structures, called pseudophores, but few regular sporangiophores. Intersexual heterokaryons have more $\beta$ carotene than comparable homokaryons whose nuclei belong all to the same sex (Murillo et al., 1978).

We have investigated the genetic requirements for the sexual activation of carotenogenesis and the relations between sexual and other stimuli. Sexual stimulation was achieved by adding

† Present address: Scripps Clinic and Research Foundation, La Jolla, Calif. 92037, USA. 
synthetic methyl trisporates or natural trisporic acids to single $(-)$ cultures of various genotypes or by constructing intersexual heterokaryons.

\section{METHODS}

The strains of Phycomyces blakesleeanus Burgeff used in this work are listed in Table 1. The auxotrophic strains A87 and B36 were given to us by Professor A. P. Eslava, Universidad de Salamanca, Spain; they require L-lysine (200 $\left.\mathrm{mg} \mathrm{l}^{-1}\right)$ and nicotinic acid $\left(1 \mathrm{mg} \mathrm{l}^{-1}\right)$, respectively. $\mathrm{C}$ strains came from the collection of the late Professor $\mathbf{M}$. Delbrück at the California Institute of Technology; the mutation mad-107 affects the late reactions of phototropism and has presumably no effect on carotenogenesis. S strains originated in this laboratory. Blakeslea trispora NRRL 2895, mating type (+), and NRRL 2896, mating type (-), and the standard $P$. blakesleeanus wildtype NRRL 1555 came from the Agricultural Research Service, Department of Argiculture, Peoria, Ill., USA. Heterokaryons are designated by placing an asterisk $(*)$ between the symbols of the constituent homokaryons.

Activated $\left(48^{\circ} \mathrm{C}, 15 \mathrm{~min}\right) P$. blakesleeanus spores were inoculated onto plates containing $25 \mathrm{ml}$ glucose minimal medium (Sutter, 1975) with L-asparagine $\mathrm{H}_{2} \mathrm{O}\left(2 \mathrm{~g} \mathrm{l}^{-1}\right)$ and agar $\left(15 \mathrm{~g} \mathrm{l}^{-1}\right)$ and incubated at $22^{\circ} \mathrm{C}$ for $4 \mathrm{~d}$ in the dark (if not otherwise stated). Experiments with heterokaryons, and their constitutive homokaryons as controls, were started with mycelial pieces, about $2 \mathrm{~mm}$ in width, taken from 2-d-old colonies. Heterokaryons were made by the microsurgical technique of Ootaki (1973).

Methyl trisporates and their analogues (Compounds I-VIII, Fig. 4) were dissolved in ethanol and spread over agar plates preinoculated and preincubated for $24 \mathrm{~h}$. Each place received $0.1 \mathrm{ml}$ ethanol. Dimethyl phthalate (dimethyl ester of 1,2-benzenedicarboxylic acid) was dissolved in ethanol and added to the molten medium before the plates were poured; the final concentrations were $2 \mathrm{mmol}$ dimethyl phthalate $\mathrm{l}^{-1}$ and $1 \%(\mathrm{v} / \mathrm{v})$ ethanol. Retinol acetate (Sigma) was dissolved in a mixture of absolute ethanol and polyoxyethylene sorbitan mono-oleate (Tween 80 , Sigma), then added to $1 \mathrm{I}$ agar medium before the plates were poured; the final concentrations were: retinol acetate, $0.75 \mathrm{mmol} \mathrm{l}^{-1}\left(250 \mu \mathrm{g} \mathrm{ml}^{-1}\right)$; ethanol, $0.1 \%(\mathrm{v} / \mathrm{v})$; and Tween $80,0.4 \%(\mathrm{v} / \mathrm{v})$.

The methods for the production, purification and assay of trisporates from mated $B$. trispora cultures were discribed by Govind \& Modi (1981) and Sutter (1970), respectively. Most of the trisporic acids used in these experiments came from cultures started with fragments of mycelia of the two sexes and grown for $6 \mathrm{~d}$ in liquid malt medium; the yield was about $15.7 \mathrm{mg}$ trisporic acids $\mathrm{l}^{-1}$. In minimal medium the yield was considerably smaller.

For the determination of carotenes, 4-d-old mycelia were peeled from the plates, freed from any visible bits of agar, and lyophilized. A weighed sample was extracted with petroleum ether (b.p. $40-60{ }^{\circ} \mathrm{C}$ ), vacuum-dried, and resuspended in $n$-hexane. $\beta$-Carotene, lycopene and phytoene were quantified from their absorption coefficients (Davies, 1965). Phytoene was previously purified chromatographically on aluminium oxide (Davies, 1965).

The results shown are means of three independent experiments, unless otherwise stated.

\section{RESULTS}

\section{Activation of the wild-type by trisporates and related compounds}

The trisporate preparation from $B$. trispora activated carotenogenesis (Fig. 1). The maximal effect was observed with $10 \mu \mathrm{g}$ trisporates $\mathrm{ml}^{-1}$; higher amounts were less effective. The dry weight of 4-d-old mycelia - $317 \pm 23 \mathrm{mg}(\mathrm{SD})$ per Petri plate - was not affected by the addition of the preparation.

Synthetic (9Z)-methyl trisporates stimulated carotene accumulation in $P$. blakesleeanus cultures of the (-) mating type (Figs 2 and 3; structural formulae and names in Fig. 4). The B form was clearly more effective than the $\mathrm{C}$ form. Little or no effect was shown by the corresponding all- $(E)$ isomers and other synthetic compounds (V-VIII) structurally related to the trisporic acids. These eight compounds were not toxic to $P$. blakesleeanus under the conditions of the experiments: the mycelial dry weight was not affected by concentrations of up to $40 \mu \mathrm{g} \mathrm{ml}^{-1}$ of any of these compounds.

While the natural trisporates from $B$. trispora stimulated carotenogenesis in both (+) and (-) wild-types, compound I did not appear to be effective on the $(+)$ strain B36 (Table 1).

\section{Activation of car mutants}

Representative strains carrying various car mutations were exposed to natural trisporates from $B$. trispora and to racemic (9Z)-methyl trisporate B (Table 1). Carotene production by the wild-type was activated by both agents, as expected from results given above. The same was found with the carS mutant. The chemoinsensitive mutants S1 19 and S144 responded to neither 


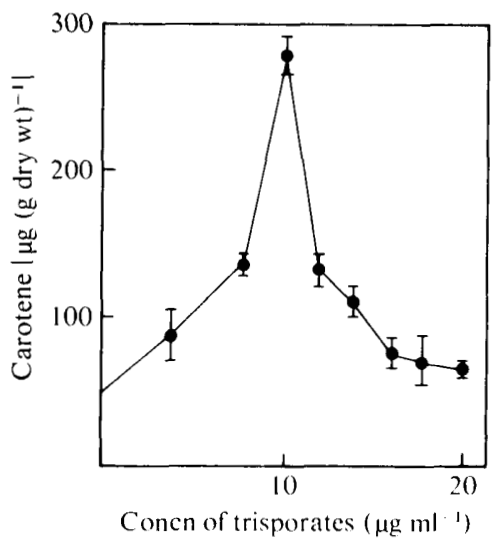

Fig. 1. Effect of trisporates from mated $B$. trispora cultures on the accumulation of $\beta$-carotene in the standard $P$. blakesleeanus wild-type. The bars indicate standard errors.
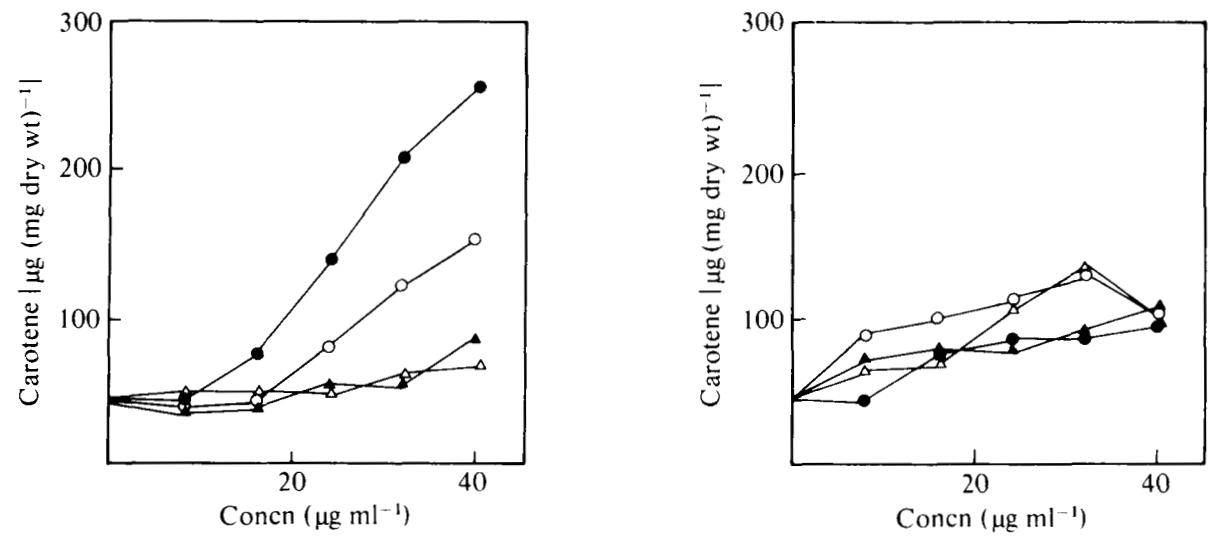

Fig. 2. Effect of methyl trisporates (compounds I-IV, see Fig. 4) on the accumulation of $\beta$-carotene in the standard. P. blakesleeanus wild-type. Compound I; $O$, compound II; $\Delta$, compound III; $\triangle$, compound IV.

Fig. 3. Effect of compounds V-VIII (see Fig. 4) on the accumulation of $\beta$-carotene in the standard $P$. blakesleeanus wild-type. $\bigcirc$, Compound V; $\bigcirc$, compound VI; $\Delta$, compound VII; $\triangle$, compound VIII.

Table 1. Carotene content of various $P$. blakesleeanus strains grown for $4 d$ in the presence of synthetic racemic (9Z)-methyl trisporate $B$ (compound $I, 40 \mu \mathrm{g} \mathrm{ml}^{-1}$ ) or natural trisporates from B. trispora $\left(10 \mu \mathrm{gl}^{-1}\right)$

The table gives the concentration ( $\mu \mathrm{g}$ per $\mathrm{g}$ dry weight) of the major carotene in each strain, which was $\beta$-carotene except in strains C9 (lycopene) and C173 (phytoene) (results marked $\dagger$ ).

Strain

NRRL 1555

$\mathrm{C} 2$

C9

C173

C115

S119

S144

S218*S219

B36

A87

B36 * A87
Genotype and references

Standard wild-type $(-)$

carA5 (-) (Murillo et al., 1981)

caR2I (-) (de la Guardia et al., 1971)

carB32 carR21 (-) (Presti et al., 1977)

carS42 mad-107 (-) (Murillo \& Cerdá-Olmedo, 1976)

carA113 (-) (Roncero \& Cerdá-Olmedo, 1982)

carI131 (-) (Roncero \& Cerdá-Olmedo, 1982)

See Murillo et al. (1978)

nicA101 (+) (Alvarez \& Eslava, 1983)

lys $A 401(-)$

nicA101 (+) *lysA401 (-)
With natural With Control trisporates compound I

$\begin{array}{rcr}54 & 296 & 300 \\ 2 & 10 & 10 \\ 1723 \dagger & 2748 \dagger & 2006 \dagger \\ 1503 \dagger & 1535 \dagger & 1447 \dagger \\ 2609 & 4277 & 4417 \\ 28 & 30 & 31 \\ 29 & 27 & 38 \\ 14062 & 14137 & 15317 \\ 52 & 105 & 38 \\ 64 & 201 & 223 \\ 269 & 252 & 249\end{array}$




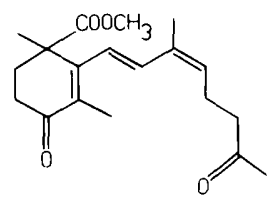

I<smiles>CC(=O)C1=C(C)C(=O)CCC1(C)C=CC(C)=CCCC(C)O</smiles>

II<smiles>CC(=O)CCC=C(C)C=CC1=C(C)C(=O)CCC1(C)C(C)=O</smiles>

III<smiles>CC(=O)C1=C(C)C(=O)CCC1(C)C=CC(C)=CCCC(C)O</smiles>

IV<smiles>CC(=O)/C=C/C1=C(C)C(=O)CCC1(C)C</smiles>

V<smiles>CC(=O)/C=C/C1=C(C)C(=O)C(OC(C)=O)CC1(C)C</smiles>

VI<smiles>CC1=CC(=O)CC(C)(C)C1(O)/C=C/C(C)=C\CO</smiles>

VII<smiles>CC(C=O)=CC=CC(C)=CCC1=C(C)CCCC1(C)C</smiles>

VIII<smiles>CC(=O)CC/C=C(C)/C=C/C1=C(C)C(=O)CC[C@@]1(C)C(=O)O</smiles>

IX<smiles>CC1=C(/C=C/C(C)=C/CC[C@H](C)O)[C@](C)(C(=O)O)CCC1=O</smiles>

$\mathrm{X}$

Fig. 4. Structural formulae of compounds used in this work: racemic (9Z)-methyl trisporate B (I), racemic $(9 Z)$-methyl trisporate $C$ (II), racemic all- $(E)$-methyl trisporate $\mathrm{B}$ (III), racemic all-( $E$ )-methyl trisporate $\mathrm{C}$ (IV), 4-oxo- $\beta$-ionone (V), racemic 3-acetoxy-4-oxo- $\beta$-ionone (VI), 4-hydroxy-4-[(3Z)-5hydroxy-3-methyl-1,3-pentadienyl]-3,5,5-trimethyl-2-cyclohexen-1-one (VII), and 8-[2,6,6-trimethylcyclohexenyll-2,6-dimethyl-octatrien(2,4,6)-al(1) (VIII). Mated B. trispora cultures contain mainly trisporates B (IX) and C (X) (Sutter et al., 1974).

Table 2. Synergism between the effects of intersexual heterokaryosis and those of light, retinol, and dimethyl phthalate on carotenogenesis

The table gives the concentration of $\beta$-carotene ( $\mu \mathrm{g}$ per g dry weight) in 4-d-old cultures. The tests of the chemicals and their controls were incubated in the dark.

\begin{tabular}{|c|c|c|c|c|c|c|}
\hline \multirow[b]{2}{*}{ Strain } & \multicolumn{2}{|c|}{ White light $\left(\mathrm{W} \mathrm{m}^{-2}\right)$} & \multicolumn{2}{|c|}{ Retinol acetate (mM) } & \multicolumn{2}{|c|}{ Dimethyl phthalate (mM) } \\
\hline & 0 & 0.43 & 0 & $0 \cdot 75$ & 0 & 2 \\
\hline B36 & 66 & 321 & 62 & 1969 & 71 & 1057 \\
\hline A 87 & 66 & 306 & 62 & 1103 & 73 & 1079 \\
\hline B36 * A87, mycelium 1 & 118 & 594 & 102 & 2894 & & \\
\hline B36 * A87, mycelium 2 & 181 & 695 & 144 & 2440 & & \\
\hline B36 * A87, mycelium 3 & 289 & 776 & 271 & 3075 & & \\
\hline B36 * A87, mycelium 4 & & & & & 260 & 1388 \\
\hline
\end{tabular}


the natural nor the synthetic compounds. The stimulation of the carotene pathway by trisporic acids seemed to require the presence of $\beta$-carotene in the receiving cells: the amount of major carotene (phytoene) of strain $\mathrm{C173}$, which is totally devoid of $\beta$-carotene, was not affected by trisporic acids, but the major carotenes of strains $C 2$ ( $\beta$-carotene) and $C 9$ (lycopene), which both produce some $\beta$-carotene, were appreciably increased.

\section{Regulation of carotenogenesis in intersexual heterokaryons}

Intersexual heterokaryons contained up to five times more $\beta$-carotene than either of the constituent homokaryons (Tables 1 and 2). The different carotene contents of different heterokaryotic mycelia depend presumably on their nuclear proportions. The intersexual heterokaryons S218 * S219 and B36 * A87 were not further stimulated by either trisporates or methyl trisporates (Table 1). Intersexual heterokaryosis thus appears to provide a saturating sexual stimulation. Cultures grown in the light or in the presence of retinol or dimethyl phthalate contained more $\beta$-carotene than those grown in the dark, irrespective of sex and intersexual heterokaryosis (Table 2). The emulsifiers needed for the application of the chemicals, present in the corresponding controls, had no effect by themselves.

\section{DISCUSSION}

Trisporates are relatively ineffective activators of carotene biosynthesis, if we compare inputs and outputs. Thus, to increase the carotene content of a Petri-dish culture from 17 to $90 \mu \mathrm{g}$ one has to add $1 \mathrm{mg}$ of the most active synthetic compound, (9Z)-methyl trisporate B (compound 1 ). The trisporate preparations from $B$. trispora produce a similar stimulation. The carotenogenic activity of the sex pheromones under physiological conditions does not appear to be very high either: mated cultures and intersexual heterokaryons (Murillo \& Cerdá-Olmedo, 1976; our results) contain about the same carotene concentrations as those obtained with exogenous trisporates.

The chemoinsensitive mutants (strains S119 and S144) are insensitive to stimulation by either synthetic methyl trisporates or natural trisporates (Table 1) and by retinol and dimethyl phthalate (unpublished). This establishes a common step in the regulation of carotenogenesis by all of these chemicals.

The sexual stimulation of carotenogenesis is additive with those induced by retinol, dimethyl phthalate, light, and mutation at gene carS. This synergism of carS mutations and sexual interaction had been observed by Murillo \& Cerdá-Olmedo (1976) and Murillo et al. (1978). The additivity indicates that at least the rate-limiting steps in the stimulation of carotenogenesis by sexual interaction are different from those of the other mechanisms of stimulation.

Sexual stimulation must thus have some common steps with the stimulation of carotenogenesis by retinol and dimethyl phthalate, but be separate from them at least at the respective limiting steps.

Particularly intriguing is the fact that the stimulatory action of trisporates apparently required $\beta$-carotene, as if the carotene itself or a product of its metabolism were involved in the chain of events leading to increased carotenogenesis. It may be that the trisporates by themselves have no effect on carotenogenesis, but only derepress the formation of trisporates from $\beta$-carotene (Bu'lock, 1976): an intermediate compound in the pathway from $\beta$-carotene to trisporate would then be the true activator of carotenogenesis.

The lack of activity of methyl trisporates on the $(+)$ strain suggests that these compounds are only effective when converted to trisporates. Methyl trisporates are believed to be made only by the $(+)$ strains and converted to trisporates by the $(-)$ strains (Sutter $\&$ Whitaker, 1981).

The inactivity of compounds V-VIII (Table 1) is somewhat surprising, in view of their resemblance to the trisporic acids and to other aromatic compounds which have turned out to be potent activators of carotenogenesis. This is only a particular case of a more general problem: the relationships between chemical structure and activation of carotenogenesis are still unclear (Cerdá-Olmedo \& Hüttermann, 1986). 
We thank Hoffmann-La Roche Co., Basel, Switzerland, and particularly Dr H. J. Mayer, for the synthetic methyl trisporates and related compounds and for other support. Mrs K. Schiedt, Dr K. Bernhard, Professor R. P. Sutter and Professor E. D. Lipson critically read the manuscript. We received a grant from the Spanish Comisión Asesora para Investigación Científica y Técnica. A group of undergraduate students (J. A. Godoy, J. Martín, M. L. Martínez, A. Mérida, I. Morales and J. J. Ortega) took part in some of the experiments.

\section{REFERENCES}

Alvarez, M. I. \& Eslava, A. P. (1983). Isogenic strains of Phycomyces blakesleeanus suitable for genetic analysis. Genetics 105, 873-879.

Austin, D. J., Bu'lock, J. D. \& Gooday, G. W. (1969). Trisporic acids: sexual hormones from Mucor mucedo and Blakeslea trispora. Nature, London 223, $1178-1179$.

Bergman, K., Eslava, A. P. \& Cerdá-Olmedo, E. (1973). Mutants of Phycomyces with abnormal phototropism. Molecular and General Genetics 123, $1-16$.

Blakeslee, A. F. (1904). Sexual reproduction in the Mucorineae. Proceedings of the National Academy of Arts and Sciences 40, 205-319.

Bu'LOCK, J. D. (1976). Hormones in fungi. In The Filamentous Fungi, vol. 2, pp. 345-368. Edited by J. E. Smith \& D. R. Berry. London: Edward Arnold.

BurgefF, H. (1914). Untersuchungen Über Variabilität, Sexualität und Erblichkeit bei Phycomyces nitens Künze. Flora (Jena) 107, 259-316.

Caglioti, L., Cainelli, G., Camerino, B., Mondelli, R., Prieto, A., Quilico, A., Salvatori, T. \& Selva, A. (1966). The structure of trisporic-C acid. Tetrahedron Supplement 7, 175-187.

Cerdí-Olmedo, E. (1985). Carotene mutants of Phycomyces. Methods in Enzymology 110A, 220-243.

Cerdá-Olmedo, E. \& Hüttermann, A. (1986). Förderung und Hemmung der Carotinsynthese bei Phycomyces durch Aromaten. Angewandte Botanik 69, $59-70$.

Cerdí-Olmedo, E. \& Torres-Martínez, S. (1979). Genetics and regulation of carotene biosynthesis. Pure and Applied Chemsitry 51, 631-637.

DAVIES, B. H. (1965). Analysis of carotenoid pigments. In Chemistry and Biochemistry of Plant Pigments, pp. 489-532. Edited by T. W. Goodwin, London: Academic Press.

Eslava, A. P., Alvarez, M. I. \& Cerdá-Olmedo, E. (1974). Regulation of carotene biosynthesis in Phycomyces by vitamin A and $\beta$-ionone. European Journal of Biochemistry 48, 617623

De la Guardia, M. D., Aragón, C. M. G., Murillo, F. J. \& CerdÁ-Olmedo, E. (1971). A carotenogenic enzyme aggregate in Phycomyces: evidence from quantitative complementation. Proceedings of the National Academy of Sciences of the United States of America 68, 2012-2015.

Govind, N. S. \& Modi, V. V. (1981). Regulation of trisporic acid synthesis in Blakeslea trispora. Indian Journal of Experimental Biology 19, 544-546.

HockING, D. (1965). Demonstration of $\beta$-carotene in gametangia of Phycomyces blakesleeanus Burgeff. Nature, London 206, 846
Jayaram, M., Presti, D. \& DelbrüCK, M. (1979). Light induced carotene synthesis in Phycomyces. Experimental Mycology 3, 42-52.

LóPEZ-DíAZ, I. \& CERDÁ-Olmedo, E. (1980). Relationship of photocarotenogenesis to other behavioural and regulatory responses in Phycomyces. Planta 150 , 134-139.

Mackinney, G., Nakayama, T., Buss, C. D. \& Chichester, C. O. (1952). Carotenoid production in Phycomyces. Journal of the American Chemical Society 74, 3456-3457.

Murillo, F. J. \& Cerdá-Olmedo, E. (1976). Regulation of carotene synthesis in Phycomyces. Molecular and General Genetics 148, 19-24.

Murillo, F. J., Calderón, I. L., López-Díaz, I. \& Cerdá-Olmedo, E. (1978). Carotene-superproducing strains of Phycomyces. Applied and Environmental Microbiology 36, 639-642.

Murillo, F. J., Torres-Martínez, S., Aragón, C. M. G. \& Cerdá-Olmedo, E. (1981). Substrate transfer in carotene biosynthesis in Phycomyces. European Journal of Biochemistry 119, 511-516.

OOTAKI, T. (1973). A new method for heterokaryon formation in Phycomyces. Molecular and General Genetics 121, 49-56.

Presti, D., Hsu Wan-Jean \& Delbrück, M. (1977). Phototropism in Phycomyces mutants lacking $\beta$ carotene. Photochemistry and Photobiology 26, 403405

Roncero, M. I. G. \& Cerdá-Olmedo, E. (1982). Genetics of carotene biosynthesis in Phycomyces. Current Genetics 5, 5-8.

SuTTER, R. P. (1970). Trisporic acid synthesis in Blakeslea trispora. Science 168, 1590-1592.

SutTER, R. P. (1975). Mutations affecting sexual development in Phycomyces blakesleeanus. Proceedings of the National Academy of Sciences of the United States of America 72, 127-130.

SutTer, R. P. (1977). Regulation of the first stage of sexual development in Phycomyces blakesleeanus and in other mucoraceous fungi. In Eucaryotic Microbes as Model Developmental Systems, pp. 251-272. Edited by D. H. O'Day \& P. A. Horgen. New York: Marcel Dekker.

SutTer, R. P. \& Whitaker, J. P. (1981). Zygophorestimulating precursors (pheromones) of trisporic acids active in (-) Phycomyces blakesleeanus; acidcatalysed anhydro-derivatives of methyl 4-dihydrotrisporate-C and 4-dihydrotrisporate-C. Journal of Biological Chemistry 256, 2334-2341.

SutTer, R. P. Harrison, T. L. \& Galasko, G. (1974). Trisporic acid biosynthesis in Blakeslea trispora via mating type-specific precursors. Journal of Biological Chemistry 249, 2282-2284. 